\title{
基于 MAX1452 的压力传感器校准系统设计
}

\section{Design of Pressure Sensor Calibration System Based on MAX1452 \\ 石晋明 付君君 赵平平 刘震 武金财}

Jinming Shi Junjun Fu Pingping Zhao Zhen Liu Xincai Wu

晋西工业集团有限责任公司

检测技术研究院

中国・山西 太原 030027

Jinxi Industrial Group Co.,Ltd. Testing

Technology Research Institute,

Taiyuan, Shanxi, 030027, China
【摘 要】文章通过对压阻式压力传感器温度漂移特点的了解, 选择 MAX1452 芯片作为信 号调理电路的核心部分, 设计了基于 MAX1452 的压力传感器校准系统。

【Abstract】This paper designs a pressure sensor calibration system based on the MAX1452 chip by understanding the temperature drift characteristics of the piezoresistive pressure sensor and selecting the MAX1452 chip as the core part of the signal conditioning circuit.

【关键词】压力; 传感器;校准; 测控

【Keywords】pressure; sensor; calibration; measurement and control 【DOI】10.36012/etr.v1i4.680

\section{1 引言}

利用型号为 MAX1452 的成本精密信号调理器对压力传 感器做数字补偿, 能够弥补传统模拟方式中补偿精度受限于 所用传感器误差的非线性特性 ${ }^{[1]}$, 以及补偿元件受温度漂移 影响的缺点。

\section{2 系统硬件的总体概况}

本设计系统主要由下位机、上位机及相应的系统应用软 件 3 部分构成。其中, 下位机部分主要由 C8051F410 和 MAX1452 智能芯片构成, 上位机部分利用 Visual C++6.0 为 软件开发工具,采用的是面向对象的方法编制上位机软件。下 位机部分主要通过 RS485 转 USB 适配器实现计算机（上位 机)的通讯功能。系统总体框图如图 1 所示。

\section{3 系统硬件选型}

系统主要由压力校准模块 (PRE)、内核模块 (MCU) 及通 信模块 (COM) 构成, 压力校准模块通过 DIO0 和 A/D0 进行通 信和数据传送, 内核模块与通信模块之间通过 TXD 和 RXD 之间进行串行通信, $\mathrm{R} / \mathrm{T}$ 端口负责片选输人或输出。接口电路 图如图 2 所示。

MAX1452 内部包含的功能模块为: 可编程的传感器激励 模块、768 字节 (6144 位)的内部 EEPROM 模块、运算放大器 模块、16 级可编程增益放大器（PGA）模块、4 个 16 位 DAC 模块及内部温度传感器模块 ${ }^{[2]}$ 。当内部温度传感器模块输出 的信号经过模数转换后, 即可用其数字量来查询 EEPROM 表格, 从此表中可以确定每一个偏移量的校准值。当其变化

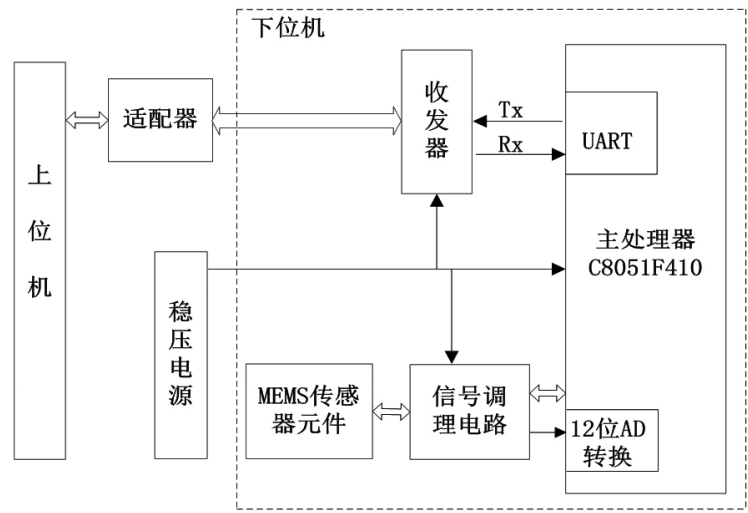

图 1 校准系统总体框图

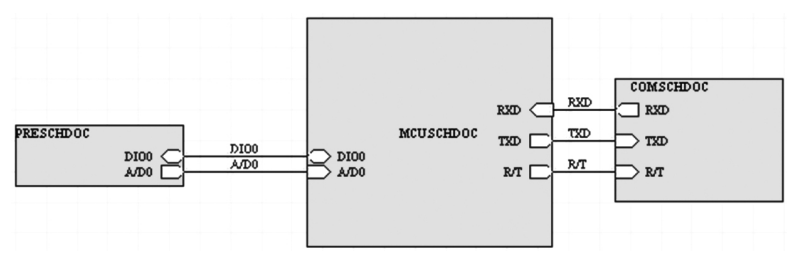

图 2 系统总体电路

范围在 $-40 \sim 125^{\circ} \mathrm{C}$ 时, 其变化精度大约为 $1.5^{\circ} \mathrm{C}$ 。每隔 $1 \mathrm{~ms}$ 时 间范围内, MAX1452 内嵌的温度传感器将给出一个在 EEPROM 中的变化值, 人们可以将查表结果传至 DAC 寄存 器, 进行数据保存。MAX1452 有 4 个 16 位的 DAC 寄存器, 用 户可以将校准系数存入 $768 \times 8 \mathrm{EEPROM}(6144$ 位 $)$ 中。此寄存 器的存储数据包含以下内容: 偏移量校准系数表、跨度校准 表、配置寄存器、偏移量温度系数寄存器、跨度温度误差修正 系数寄存器。

110 2019 年 12 月 
为了实现对 MAX1452 的控制，需将其锁定引脚 (UNLOCK) 接入高电平, 旨在实现数字接口 DIO (Digital Input Output)的双向通信功能。然后, 需要在上位机的软件中输入 失调 FSO 及温度补偿的校准精度等参数, 输入参数后选择需 要配置的 EEPROM 单元地址及寄存器种类, 之后将所需指令 发送至下位机, 通过 MAX1452 与下位机的通讯过程,进而完 成指令传输。最后, 按照校准的具体过程调整温度值与压力 值, 将校准过程的输出值进行记录, 通过在多温度条件下, 对 各个压力点进行测量, 将得到的输出值进行曲线拟合, 从而完 成对压力传感器的计量校准。

\section{4 系统的软件开发}

在本次设计的校准系统中，下位机部分的通信方式是通 过 RS485 转 USB 适配器与上位机进行对接, 串行通信的具体 格式是: 1 位作为启动位, 8 位作为数据位, 1 位作为停止位, 不设立校验位, 串行通信的速率为 $1 \mathrm{Mbps}$ 。

在本系统中, 采用的通信方式为半双工异步的方式 ${ }^{[3]}$, 系 统时钟频率根据具体需求设置为 $25.4 \mathrm{MHz}$, 下位机的工作步 骤就是首先进行系统的初始化, 此时进入等待上位机指令的 监听状态, 上位机在发出指令后, 下位机及单片机通过对此指 令进行解析, 然后将解析后的指令发送至 MAX1452, 发送指 令完毕后, 下位机向上位机返回回复指令 “ $O K ” 。$ 此时, 需要返 回的数据则在上诉指令发送完毕后, 发送给上位机。具体流程 如图 3 所示。

\section{5 校准过程与标定实验结果}

文章以压力传感器的校准过程为例, 说明应用 MAX1452 进行压力校准及补偿的具体方法与步骤。MAX1452 的模拟部 分是由激励传感器桥的所有信号成分组成的, 通过将传感器 的微小信号进行放大，为校准过程提供尽可能多的可选模拟 通道。通过改变可编程放大器的增益及偏移量的大小, 同时, 改变传感器电桥上的激励电压、电流值, 实现对压力传感器的 校准与修正。

作为压力传感器的静态参数校准项目, 零点和灵敏度的 校准是重中之重, 这关系到压力传感器计量特性的准确性, 论 文提出的方法较之前简化了很多，而且高效准确。EEPROM 通过查找表格里提供的对压力传感器的灵敏度和偏置 DAC 的标准值, 可以有效地将各个校准点相互区别开来。 MAX1452 还具备高度的可伸缩性和可见性, 使具体的校准过 程能够高效快速地完成。

在利用 MAX1452 进行压力传感器校准时, 具体工作主

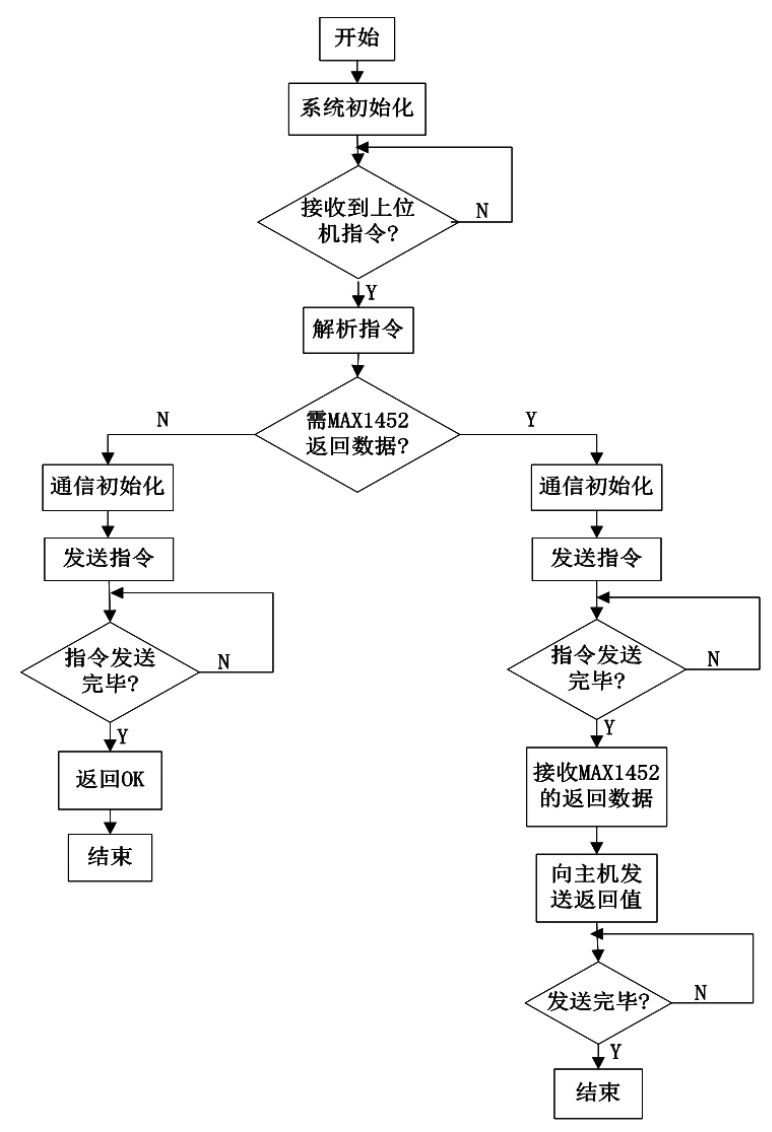

图 3 下位机软件流程图

要包含两大方面,初始校准及多点温度校准。不同的压力传感 器其生产厂家不尽相同, 这就导致传感器的桥路电阻、灵敏度 等很多参数是不相同的。在使用前, 由于计量特性的要求, 需 要在常温下进行初始校准, 这不仅需要保证 MAX1452 内部 的电路工作在线性可调节的范围内，也要满足为传感器设定 初始参数的要求。

\section{6 结语}

论文通过对压阻式压力传感器温度漂移特点的了解, 选 择 MAX1452 芯片作为信号调理电路的核心部分，设计了基 于 MAX1452 的压力传感器校准系统, 进行了压力传感器的 温度补偿试验。通过试验结果的比较,验证了校准系统对于压 力传感器的压力参数测量精度优于 $0.5 \% \mathrm{FS}$ 。

\section{参考文献}

[1]章诵兰.物联网高速发展国内传感器迎来黄金发展期[J].自动 化与仪表,2012(8):56.

[2]王定贤,尹晓利,赵建伟,等.基于 VB 的计算机与智能仪器串口 通信[J].甘肃联合大学学报(自然科学版),2011(4):71-74.

[3] 睢毅.曲线拟合在压力传感器中的研究与实现 [J].中国医疗器 械信息,2011(4):34-36+43. 